\title{
Article
}

\section{Risk Due to ABO Incompatibility and Donor-Recipient Weight Mismatch in Living Donor Kidney Transplantation: A National Cohort Study}

\author{
Jun Young Lee ${ }^{1,2,+} \oplus$, Sung Hwan Cha ${ }^{3,+}$, Sung Hwa Kim ${ }^{4}{ }^{\infty}$, Kyung Hwan Jeong ${ }^{5}$, Ku Yong Chung ${ }^{6}$,

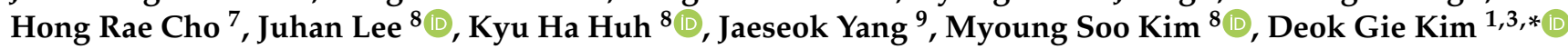 \\ and on behalf of the Korean Organ Transplantation Registry Study Group $\ddagger$
}

check for updates

Citation: Lee, J.Y.; Cha, S.H.; Kim, S.H.; Jeong, K.H.; Chung, K.Y.; Cho, H.R.; Lee, J.; Huh, K.H.; Yang, J.; Kim, M.S.; et al. Risk Due to ABO Incompatibility and Donor-Recipient Weight Mismatch in Living Donor Kidney Transplantation: A National Cohort Study. J. Clin. Med. 2021, 10, 5674. https://doi.org/10.3390/ jcm10235674

Academic Editor: Kent Doi

Received: 5 November 2021 Accepted: 30 November 2021 Published: 1 December 2021

Publisher's Note: MDPI stays neutral with regard to jurisdictional claims in published maps and institutional affiliations.

Copyright: (c) 2021 by the authors. Licensee MDPI, Basel, Switzerland. This article is an open access article distributed under the terms and conditions of the Creative Commons Attribution (CC BY) license (https:// creativecommons.org/licenses/by/ $4.0 /)$.
1 Transplantation Center, Wonju Severance Christian Hospital, Wonju 26426, Korea; junyoung07@yonsei.ac.kr 2 Department of Nephrology, Yonsei University Wonju College of Medicine, Wonju 26426, Korea

3 Department of Surgery, Yonsei University Wonju College of Medicine, Wonju 26426, Korea; luvnya82@yonsei.ac.kr

4 Department of Biostatistics, Yonsei University Wonju College of Medicine, Wonju 26426, Korea; juniver1057@naver.com

5 Department of Internal Medicine, Kyung Hee University College of Medicine, Seoul 02447, Korea; khjeong@khu.ac.kr

6 Department of Surgery, Ewha Womans University Mokdong Hospital, Seoul 07985, Korea; kuyong@ewha.ac.kr

7 Department of Surgery, Ulsan University Hospital, Ulsan 44030, Korea; hrcho@uuh.ulsan.kr

8 Department of Surgery, Yonsei University College of Medicine, Seoul 03722, Korea; laplaine@yuhs.ac (J.L.); khhuh@yuhs.ac (K.H.H.); ysms91@yuhs.ac (M.S.K.)

9 Department of Internal Medicine, Yonsei University College of Medicine, Seoul 03722, Korea; jcyj@@snu.ac.kr

* Correspondence: exdelcomp20@gmail.com; Tel.: +82-33-741-0510

+ These authors contributed equally to this work.

$\ddagger$ Membership of the Korean Organ Transplantation Registry Study Group is provided in the Acknowledgments.

Abstract: The effect of donor-recipient weight mismatch is not well established in ABO-incompatible living donor kidney transplantation (LDKT). A total of 2584 LDKT patients in the Korean Organ Transplantation Registry were classified into four groups according to the presence or absence of ABO incompatibility and donor-recipient weight mismatch (donor-to-recipient weight ratio (DRWR) $<0.8$ ). In a multivariable Cox analysis, the combination of ABO incompatibility and DRWR incompatibility $(n=124)$ was an independent risk factor for graft survival $(\mathrm{HR}=2.73,95 \% \mathrm{CI}=1.11-6.70)$ and patient survival $(\mathrm{HR}=3.55,95 \% \mathrm{CI}=1.39-9.04)$, whereas neither factor alone was a significant risk factor for either outcome. The combination of ABO incompatibility and DRWR incompatibility was not an independent risk factor for biopsy-proven graft rejection $(\mathrm{HR}=1.27,95 \% \mathrm{CI}=0.88-1.82)$; however, it was an independent risk factor for pneumonia ( $\mathrm{HR}=2.94,95 \% \mathrm{CI}=1.64-5.57)$. The mortality rate due to infection was higher among patients with both $\mathrm{ABO}$ incompatibility and DRWR incompatibility than among patients with neither factor or with either factor alone. The combination of ABO incompatibility and DRWR incompatibility was an independent risk factor for graft and patient survival after LDKT, whereas neither factor alone significantly affected graft or patient survival. Thus, donor-recipient weight matching should be cautiously considered in LDKT with $\mathrm{ABO}$ incompatibility.

Keywords: ABO incompatible; kidney transplantation; living donor; weight mismatch

\section{Introduction}

Kidney transplantation (KT) is the best treatment for most patients with end-stage renal disease [1]. Amidst the circumstance of organ shortage, ABO-incompatible living 
donor KT (ABOi LDKT) is one way to expand the donor pool. Despite excellent outcomes of ABOi LDKT in an early study [2], there have been conflicting reports regarding the safety of ABOi LDKT compared to that of ABO-compatible living donor KT (ABOc LDKT) [3-9]. Furthermore, a recent meta-analysis found that ABOi LDKT resulted in lower short-term graft and patient survival compared with ABOc LDKT [10]. In contrast, a recent well-designed cohort study demonstrated that patients who underwent ABOi LDKT had improved survival compared with patients that waited for a transplant either from a deceased donor or an $\mathrm{ABO}$-compatible live donor [11]. In this context, further risk analysis of ABOi LDKT in comparison with ABOc LDKT is warranted.

Nephron mass is an important determinant of the fate and function of the kidney [12], as insufficient nephron mass is thought to be the starting point of hyperfiltration, which ultimately leads to renal allograft damage $[13,14]$. Given that kidney size is associated with patient weight [15], several studies have emphasized the negative effect of low donor-torecipient weight ratio (DRWR) on KT outcomes [16-19]. It is not known, however, if weight mismatch between donor and recipient interacts with $\mathrm{ABOi}$ when both risk factors are present. Therefore, we aimed to investigate whether the combination of ABOi and DRWR incompatibility (DRWRi) is an independent risk factor in living donor KT (LDKT).

\section{Materials and Methods}

\subsection{Study Population}

We conducted a retrospective cohort study of KT recipients in the Korean Organ Transplantation Registry (KOTRY) who underwent KT in Korea between May 2014 and December 2018. Details of the KOTRY are described elsewhere [20]. A total of 3050 LDKTs were in the KOTRY at the time of our study. We excluded cases with zero-human leukocyte antigen (HLA) mismatch $(n=209)$, crossmatch-positive LDKT $(n=252)$, or lack of data $(n=5)$. The remaining 2584 LDKTs included 1941 ABOc LDKTs and 643 ABOi LDKTs. We defined DRWRi as a DRWR less than 0.8, which was near the cutoff for the 25th percentile among all LDKTs in the dataset; we defined DRWR compatibility (DRWRc) as a DRWR greater than 0.8 (Figure S1). For our analysis, we divided the patients into four groups depending on the presence or absence of ABOc and DRWRc: ABOc-DRWRc $(n=1570)$, ABOc-DRWRi $(n=371)$, ABOi-DRWRc $(n=519)$, and ABOi-DRWRi $(n=124$; Figure 1$)$.

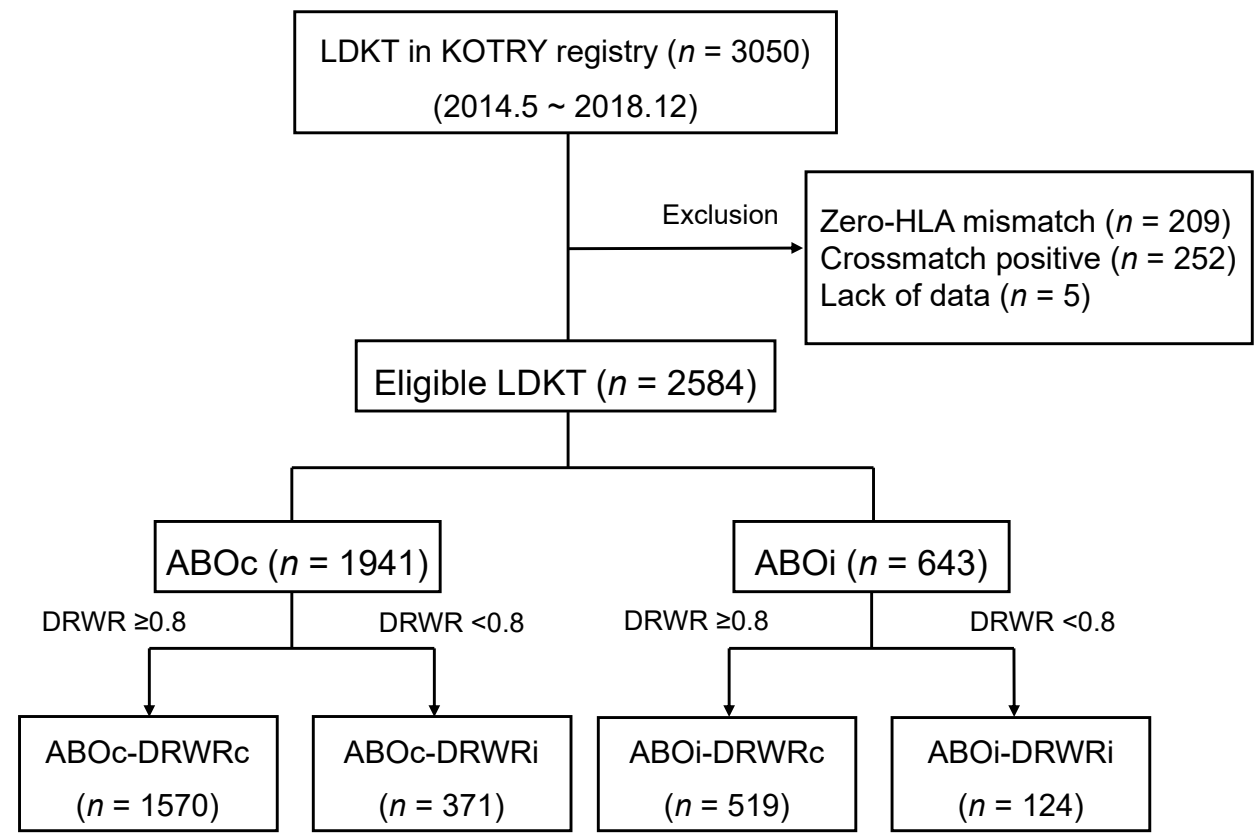

Figure 1. Study population. DRWR, donor-recipient weight ratio; HLA, human leukocyte antigen; LDKT, living donor kidney transplantation. 


\subsection{Data Collection}

We retrieved data on the demographics of the recipients and donors, underlying kidney disease, donor-specific antibody (DSA) positivity, and induction agent. Serum creatinine was measured at the time of discharge, at 6 and 12 months, and annually thereafter. The estimated glomerular filtration rate (eGFR) was calculated from the serum creatinine level using the Modification of Diet in Renal Disease equation [21], which assigns a value of zero when graft failure occurs. Detailed data regarding ABOi LDKT, such as anti-blood group antibody and pretransplant desensitization, were unavailable for more than half of the sample, so we did not include them in our analysis (the available data are listed in Table S1).

The causes of graft failure and patient death were recorded. We defined graft failure as a need for return to dialysis or re-transplantation. Data from patients who died with a functioning graft were censored in the analysis of graft failure. The first event of biopsyproven rejection (BPAR) was recorded for each patient. The causative pathogens and infection sites were recorded for infections that required re-admission to the hospital after KT. The primary end point of the study was death-censored graft survival. Secondary end points were patient survival, BPAR, and infection.

\subsection{Statistical Analysis}

Categorical variables were compared by Chi-square test or Fisher's exact test and presented as the number (proportion). Continuous variables were compared by one-way analysis of variance (ANOVA) and presented as the mean \pm standard deviation. Survival curves were generated using the Kaplan-Meier method and compared by log-rank test. Cox regression analysis was performed for outcomes adjusted with covariates for which the $p$-value was $<0.10$ in a univariable Cox analysis. A multivariable model for patient survival was established by stepwise regression to ensure statistical significance with limited number of events. The results were presented as hazard ratios (HRs) with 95\% confidence intervals (CIs). All analyses were performed using standard software (SPSS v23.0; IBM, Armonk, NY, USA, and R freeware v3.6.3, R Foundation for Statistical Computing, Vienna, Austria). $p<0.05$ was considered statistically significant.

\section{Results}

\subsection{Baseline Characteristics}

The demographics of the study sample are shown in Table 1. The mean recipient age ranged from 47.1 to 49.8 years, with no significant difference among the four groups. Male recipients and female donors were predominant in the ABOc-DRWRi and ABOi-DRWRi groups, reflecting weight differences between the sexes. The body mass index (BMI) of the recipients was naturally higher in the two DRWRi groups than in the DRWRc groups, whereas that of the donors was vice versa. Genetically unrelated donors, kidney donation from a spouse, and HLA mismatch were all more common in the ABOi-DRWRi group than in the other three groups (Table 1). The mean duration of pre-transplant dialysis was similar among the four groups, ranging from 11.5 months to 17.8 months. Re-transplantation tended to be more frequent in the two DRWRc groups than in the DRWRi groups. Diabetes, hypertension, and cardiovascular disease were generally more frequent in the two DRWRi groups, reflecting the male predominance in these groups. The frequency of pre-transplant positivity for DSA with negativity for lymphocyte crossmatch (XM-DSA+) was $5.4 \%$ vs. $1.6 \%$ vs. $9.6 \%$ vs. $6.5 \%$ in the ABOc-DRWRc, ABOc-DRWRi, ABOi-DRWRc, and ABOiDRWRi groups, respectively. Most patients used the IL-2 receptor antibody as an induction agent, although some in each group used anti-thymocyte globulin. 
Table 1. Baseline characteristics.

\begin{tabular}{|c|c|c|c|c|c|}
\hline Variables & $\begin{array}{l}\text { ABOc-DRWRc } \\
(n=1570)\end{array}$ & $\begin{array}{c}\text { ABOc-DRWRi } \\
(n=371)\end{array}$ & $\begin{array}{l}\text { ABOi-DRWRc } \\
\quad(n=519)\end{array}$ & $\begin{array}{l}\text { ABOi-DRWRi } \\
\quad(n=124)\end{array}$ & $p$ \\
\hline Age, years & $47.6 \pm 12.2$ & $47.1 \pm 10.7$ & $47.6 \pm 12.5$ & $49.8 \pm 9.2$ & 0.192 \\
\hline Sex, male & $847(53.9)$ & $345(93.0)$ & $277(53.4)$ & $116(93.5)$ & $<0.001$ \\
\hline BMI, $\mathrm{kg} / \mathrm{m}^{2}$ & $22.5 \pm 3.3$ & $26.6 \pm 3.4$ & $22.3 \pm 3.3$ & $26.3 \pm 3.6$ & $<0.001$ \\
\hline Donor age, years & $45.6 \pm 12.2$ & $48.0 \pm 9.9$ & $47.0 \pm 12.1$ & $47.6 \pm 9.7$ & 0.001 \\
\hline Donor sex, male & $804(51.2)$ & $30(8.1)$ & $261(50.3)$ & $8(6.5)$ & $<0.001$ \\
\hline Donor BMI, $\mathrm{kg} / \mathrm{m}^{2}$ & $24.7 \pm 3.1$ & $22.2 \pm 2.4$ & $24.5 \pm 3.2$ & $22.1 \pm 2.3$ & $<0.001$ \\
\hline Recipient-donor relationship & & & & & $<0.001$ \\
\hline Unrelated & $596(38.0)$ & $213(57.4)$ & $278(53.6)$ & $98(79.0)$ & \\
\hline Related & $974(62.0)$ & $158(42.6)$ & $241(46.4)$ & $26(21.0)$ & \\
\hline Recipient-donor relationship-detail & & & & & $<0.001$ \\
\hline Spouse & $487(31.0)$ & $195(52.6)$ & $248(47.8)$ & $91(73.4)$ & \\
\hline Parent & $280(17.8)$ & $61(16.4)$ & $91(17.5)$ & $10(8.1)$ & \\
\hline Offspring & $345(22.0)$ & $28(7.5)$ & $79(15.2)$ & $7(5.6)$ & \\
\hline Sibling & $349(22.3)$ & $69(18.6)$ & $71(13.7)$ & $9(7.3)$ & \\
\hline Other unrelated & $109(6.9)$ & $18(4.9)$ & $30(5.8)$ & $7(5.6)$ & \\
\hline $\begin{array}{l}\text { Number of HLA mismatch } \\
\text { (for A/B/DR) }\end{array}$ & $3.6 \pm 1.4$ & $3.9 \pm 1.5$ & $3.9 \pm 1.5$ & $4.3 \pm 1.4$ & $<0.001$ \\
\hline Cause of ESRD & & & & & 0.001 \\
\hline Diabetes & $343(21.8)$ & $120(32.3)$ & $126(24.3)$ & $40(32.3)$ & \\
\hline Hypertension & $214(13.6)$ & $51(13.7)$ & $61(11.8)$ & $21(16.9)$ & \\
\hline Glomerular disease & $568(36.2)$ & $100(27.0)$ & $181(34.9)$ & $33(26.6)$ & \\
\hline PCKD & $74(4.7)$ & $22(5.9)$ & $30(5.8)$ & $8(6.5)$ & \\
\hline Other disease & $48(3.1)$ & $4(1.1)$ & $19(3.7)$ & $3(2.4)$ & \\
\hline Unknown & $323(20.6)$ & $74(19.9)$ & $102(19.7)$ & $19(15.3)$ & \\
\hline Dialysis duration, months & $17.8 \pm 35.7$ & $14.5 \pm 34.5$ & $17.7 \pm 36.3$ & $11.5 \pm 23.4$ & 0.110 \\
\hline Retransplantation & $114(7.3)$ & $7(1.9)$ & $28(5.4)$ & $3(2.4)$ & $<0.001$ \\
\hline Diabetes & $441(28.1)$ & $147(39.6)$ & $162(31.2)$ & $53(42.7)$ & $<0.001$ \\
\hline Hypertension & $1388(88.4)$ & $348(93.8)$ & $467(90.0)$ & $121(97.6)$ & $<0.001$ \\
\hline CVD & $134(8.5)$ & $48(12.9)$ & $43(8.3)$ & $13(10.5)$ & 0.050 \\
\hline XM-DSA+ & $84(5.4)$ & $6(1.6)$ & $50(9.6)$ & $8(6.5)$ & $<0.001$ \\
\hline Induction agent & & & & & 0.330 \\
\hline IL-2 receptor antibody & $1390(88.5)$ & $339(91.4)$ & $463(89.2)$ & 107 (86.3) & \\
\hline Anti-thymocyte globulin & $180(11.5)$ & $32(8.6)$ & $56(10.8)$ & $17(13.7)$ & \\
\hline
\end{tabular}

BMI, body mass index; CVD, cardiovascular disease; DSA, donor specific antibody; ESRD, end stage renal disease; HLA, human leukocyte antigen; IL, interleukin; PCKD, polycystic kidney disease; XM, crossmatch.

\subsection{Graft Function}

Figure 2 shows the mean eGFR among the four groups from the time of hospital discharge to 48 months after KT. At discharge, the mean eGFR was lower in the ABOcDRWRi $\left(59.0 \mathrm{~mL} / \mathrm{min} / 1.73 \mathrm{~m}^{2}\right)$ and ABOi-DRWRi $\left(57.8 \mathrm{~mL} / \mathrm{min} / 1.73 \mathrm{~m}^{2}\right)$ groups than in the ABOc-DRWRc $\left(75.3 \mathrm{~mL} / \mathrm{min} / 1.73 \mathrm{~m}^{2}\right)$ and ABOi-DRWRc $\left(75.2 \mathrm{~mL} / \mathrm{min} / 1.73 \mathrm{~m}^{2}\right)$ groups. This trend was maintained throughout the study period. The full results are provided in Table S2.

\subsection{Graft Survival}

During a mean follow-up of $36.2 \pm 15.7$ months, graft loss occurred in $50(1.9 \%)$ patients. Rejection was the most common cause of graft loss $(n=23,0.9 \%)$, the detailed causes of which are presented in Figure S2. In the Kaplan-Meier analysis, death-censored graft survival was lower in the ABOi-DRWRi group than in the other groups, although the difference was not statistically significant $(p=0.063$, Figure 3a). The cumulative incidence of graft loss at 5 years was $2.7 \%$ vs. $3.0 \%$ vs. $2.3 \%$ vs. $5.0 \%$ in the ABOc-DRWRc, ABOc-DRWRi, ABOi-DRWRc, and ABOi-DRWRi groups, respectively. In univariable and multivariable Cox regression analyses (Table 2), ABOi and DRWRi were not independent risk factors for graft survival; however, the combination of both factors was $(\mathrm{HR}=2.73$, $95 \% \mathrm{CI}=1.11-6.70, p=0.028)$, together with other factors such as age and the number of 
HLA mismatches. The full results of the Cox regression analyses of death-censored graft survival are provided in Table S3.

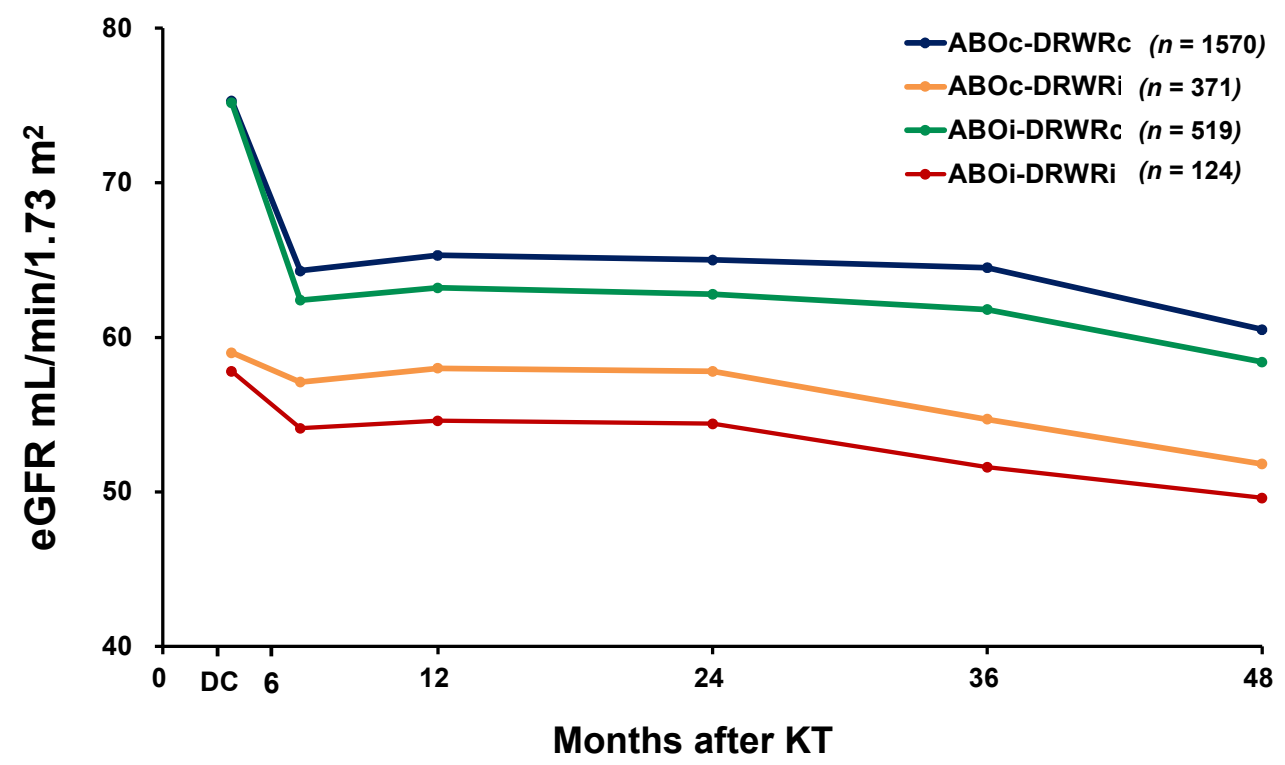

Figure 2. Graft function from hospital discharge to 48 months after KT. $p$-values of ANOVA and pos hoc analyses at each time point are provided in the Table S2. DC, discharge; KT, kidney transplantation.


(b)

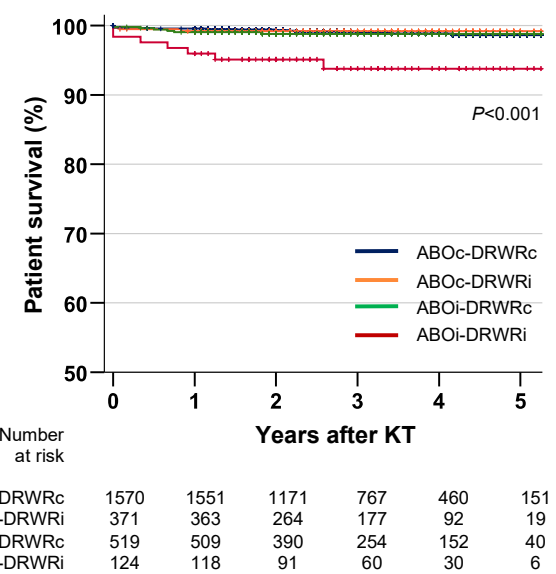

(d)

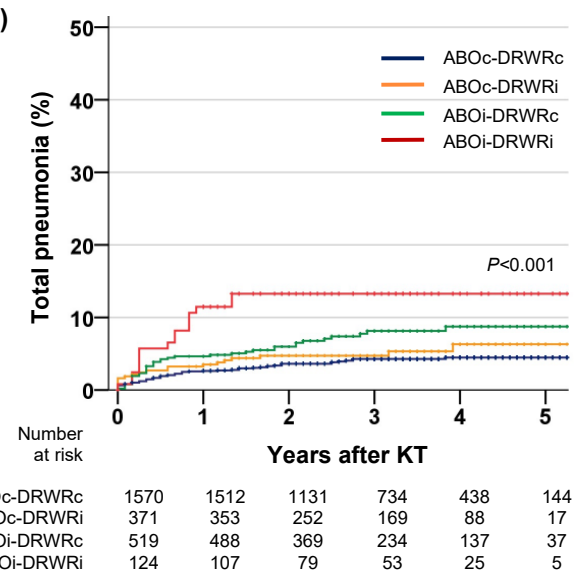

Figure 3. Kaplan-Meier analyses of the outcomes. (a) Death-censored graft survival, (b) patient survival, (c) BPAR, and (d) total pneumonia. BPAR, biopsy-proven acute rejection; KT, kidney transplantation. 
Table 2. Univariable and multivariable Cox analysis.

\begin{tabular}{|c|c|c|c|c|}
\hline \multirow[b]{2}{*}{ Variables } & \multicolumn{2}{|l|}{ Univariable Cox } & \multicolumn{2}{|c|}{ Multivariable Cox } \\
\hline & HR $(95 \%$ CI $)$ & $p$ & HR $(95 \%$ CI) & $p$ \\
\hline \multicolumn{5}{|c|}{ For death-censored graft survival a } \\
\hline ABOi vs. ABOc & $1.18(0.64-2.19)$ & 0.601 & $1.10(0.59-2.04)$ & 0.770 \\
\hline DRWRi vs. DRWRc & $1.87(1.02-3.43)$ & 0.042 & $1.75(0.95-3.23)$ & 0.074 \\
\hline ABOc-DRWRc & Reference & & Reference & \\
\hline ABOc-DRWRi & $1.46(0.69-3.10)$ & 0.327 & $1.32(0.62-2.83)$ & 0.469 \\
\hline ABOi-DRWRc & $0.90(0.41-1.98)$ & 0.791 & $0.82(0.37-1.81)$ & 0.624 \\
\hline ABOi-DRWRi & $2.94(1.21-7.12)$ & 0.017 & $2.73(1.11-6.70)$ & 0.028 \\
\hline \multicolumn{5}{|l|}{ For patient survival ${ }^{b}$} \\
\hline ABOi vs. ABOc & $2.18(1.07-4.45)$ & 0.032 & $1.65(0.79-3.42)$ & 0.181 \\
\hline DRWRi vs. DRWRc & $2.05(0.96-4.34)$ & 0.063 & $1.64(0.75-3.59)$ & 0.214 \\
\hline ABOc-DRWRc & Reference & & Reference & \\
\hline ABOc-DRWRi & $0.86(0.25-2.97)$ & 0.813 & $0.67(0.19-2.35)$ & 0.533 \\
\hline ABOi-DRWRc & $1.21(0.47-3.12)$ & 0.693 & $0.93(0.35-2.42)$ & 0.875 \\
\hline ABOi-DRWRi & $6.02(2.46-14.77)$ & $<0.001$ & 3.55 (1.39-9.04) & 0.008 \\
\hline \multicolumn{5}{|l|}{ For BPAR $^{\mathrm{c}}$} \\
\hline ABOi vs. ABOc & $1.24(1.03-1.50)$ & 0.024 & $1.14(0.94-1.38)$ & 0.190 \\
\hline DRWRi vs. DRWRc & $1.31(1.07-1.60)$ & 0.009 & $1.05(0.84-1.31)$ & 0.661 \\
\hline ABOc-DRWRc & Reference & & Reference & \\
\hline ABOc-DRWRi & $1.29(1.02-1.64)$ & 0.037 & $1.02(0.79-1.32)$ & 0.879 \\
\hline ABOi-DRWRc & $1.23(0.99-1.52)$ & 0.060 & $1.11(0.89-1.38)$ & 0.355 \\
\hline ABOi-DRWRi & $1.66(1.17-2.34)$ & 0.004 & $1.27(0.88-1.82)$ & 0.203 \\
\hline \multicolumn{5}{|l|}{ For pneumonia ${ }^{d}$} \\
\hline ABOi vs. ABOc & $2.08(1.47-2.93)$ & $<0.001$ & $2.02(1.42-2.87)$ & $<0.001$ \\
\hline DRWRi vs. DRWRc & $1.53(1.04-2.25)$ & 0.030 & $1.28(0.85-1.94)$ & 0.235 \\
\hline ABOc-DRWRc & Reference & & Reference & \\
\hline ABOc-DRWRi & $1.34(0.80-2.25)$ & 0.262 & 1.15 (0.67-1.97) & 0.605 \\
\hline ABOi-DRWRc & $1.91(1.27-2.86)$ & 0.002 & $1.87(1.24-2.82)$ & 0.003 \\
\hline ABOi-DRWRi & $3.53(2.04-6.12)$ & $<0.001$ & $2.94(1.64-5.57)$ & $<0.001$ \\
\hline
\end{tabular}

Models for each outcome were determined with covariates of which $p$-value was $<0.10$ in univariable Cox. Full results of Cox analysis were provided in Supplement Tables S2-S4. ${ }^{\text {a }}$ Multivariable Cox model for death-censored graft survival included age, donor age, number of HLA mismatches, and XM-DSA+. ${ }^{\mathrm{b}}$ Multivariable Cox model for patient survival was established by stepwise regression for the Optimal statistical significance with limited number of death events $(n=30)$. CVD, unrelated donor and XM-DSA+ were finally included in the model. ${ }^{c}$ Multivariable Cox model for BPAR included age, sex, retransplantation, CVD, donor age, donor sex, donor BMI, number of HLA mismatches, XM-DSA+, and induction agent. ${ }^{\mathrm{d}}$ Multivariable Cox model for pneumonia included age, sex, CVD, donor age, and unrelated donor. BPAR, biopsy-proven acute rejection; CVD, cardiovascular disease; DSA, donor specific antibody; HLA, human leukocyte antigen; $\mathrm{XM}$, crossmatch.

\subsection{Patient Survival}

Thirty-one (1.2\%) patients died during the study period. Infection was the most common cause of death $(n=16,0.6 \%)$, followed by unknown causes $(n=7,0.3 \%)$ and cardiovascular disease $(n=4,0.2 \%)$. The detailed causes of death are presented in Figure S3. In the Kaplan-Meier analysis, patient survival was significantly lower in the ABOi-DRWRi group than in the other three groups $(p<0.001$, Figure $3 b)$. The cumulative mortality at 5 years was $1.4 \%, 0.8 \%, 1.2 \%$, and $6.2 \%$ in the ABOc-DRWRc, ABOc-DRWRi, ABOi-DRWRc, and ABOi-DRWRi groups, respectively. In the Cox regression analysis (Table 2), ABOi and DRWRi were not independent risk factors for patient survival; however, the combination of both factors was ( $\mathrm{HR}=3.55,95 \% \mathrm{CI}=1.39-9.04, p=0.008)$, along with other factors such as cardiovascular disease, unrelated donor, and XM-DSA+ (Table S4).

\subsection{Biopsy-Proven Acute Rejection}

In the Kaplan-Meier analysis, BPAR was more common in the ABOi-DRWRi group than in the other groups ( $p=0.006$, Figure 3c), although after adjustment with other covariates, ABOi-DRWRi ( $\mathrm{HR}=1.27,95 \% \mathrm{CI}=0.88-1.82, p=0.203)$, ABOi $(\mathrm{HR}=1.14$, $95 \% \mathrm{CI}=0.95-1.38, p=0.190)$, and DRWRi (HR $=1.05,95 \% \mathrm{CI}=0.84-1.31, p=0.661)$ were not independent risk factors for BPAR (Table 2). The independent risk factors for 
BPAR were age, male sex, re-transplantation, donor age, number of HLA mismatches, and XM-DSA+ (Table S5). For patients who did not experience BPAR, graft survival was similar among the four groups ( $p=0.563$, Figure 4a) Among patients who experienced BPAR, graft survival was lower in the ABOi-DRWRi group than in the other groups, although the difference was not statistically significant $(p=0.135$, Figure $4 b)$.
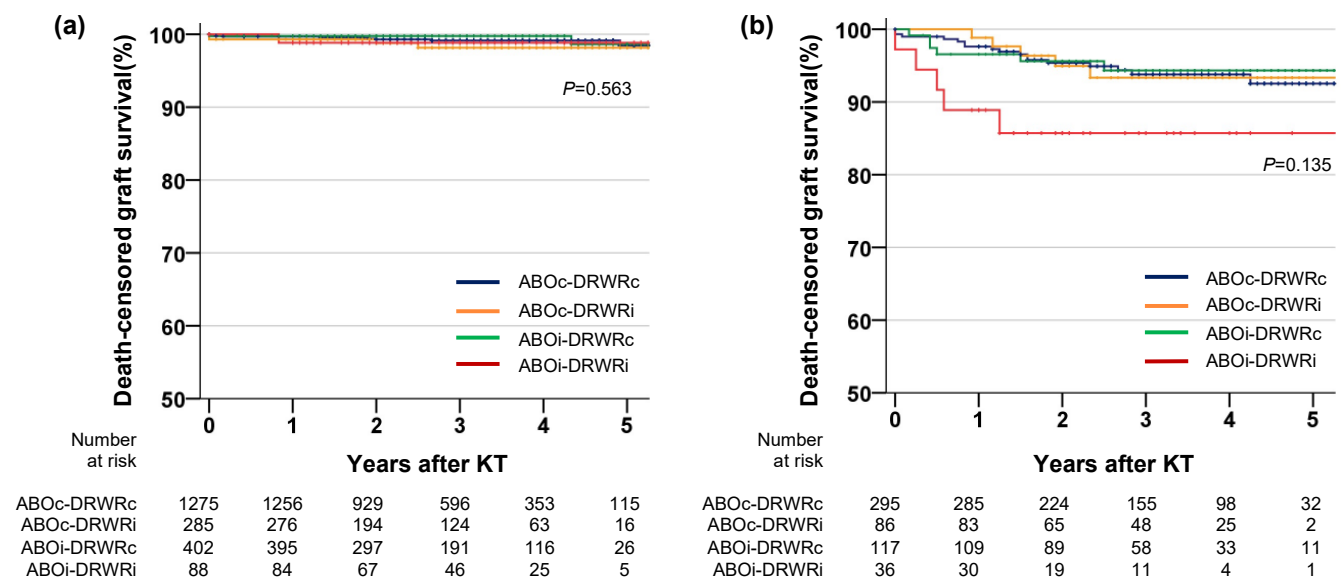

Figure 4. Graft survival among patients who did or did not experience BPAR. (a) Did not experienced BPAR, (b) experienced BPAR. BPAR, biopsy-proven acute rejection; KT, kidney transplantation.

\subsection{Infection}

We compared the incidences of infections that required hospitalization within 1 year after KT (Table 3) and found no significant differences in the overall incidence of infection or the incidences of urinary tract infection, bacteremia, viral infection, and fungal infection among the four groups. In contrast, bacterial pneumonia, viral pneumonia, and Pneumocystis jiroveci pneumonia occurred more frequently in the ABOi-DRWRi group than in the other groups (Table 3). Kaplan-Meier analyses showed that cumulative incidence of pneumonia was significantly higher in the ABOi-DRWRi group than in the other groups $(p<0.001$, Figure 3d). In univariable and multivariable Cox analyses of total pneumonia events during the study period, the combination of ABOi and DRWRi was an independent risk factor $(\mathrm{HR}=2.94,95 \% \mathrm{CI}=1.64-5.57, p<0.001)$, as were ABOi with DRWRc $(\mathrm{HR}=1.87$, $95 \% \mathrm{CI}=1.24-2.82, p=0.003)$ and $\mathrm{ABOi}$ alone $(\mathrm{HR}=2.02,95 \% \mathrm{CI}=1.42-2.87, p<0.001$; Table S6). The mortality rate due to infection during the study period was higher in the ABOi-DRWRi group than in the other groups, although the rate was low in all groups $(0.4 \%, 0.5 \%, 1.0 \%$, and $2.4 \%$ in the ABOc-DRWRc, ABOc-DRWRi, ABOi-DRWRc, and ABOi-DRWRi groups, respectively, $p=0.033$ ).

Table 3. Infectious complications within 1 year after kidney transplantation.

\begin{tabular}{|c|c|c|c|c|c|}
\hline Variables & $\begin{array}{c}\text { ABOc-DRWRc } \\
(n=1570)\end{array}$ & $\begin{array}{c}\text { ABOc-DRWRi } \\
\quad(n=371)\end{array}$ & $\begin{array}{c}\text { ABOi-DRWRc } \\
(n=519)\end{array}$ & $\begin{array}{l}\text { ABOi-DRWRi } \\
\quad(n=124)\end{array}$ & $p$ \\
\hline Total infections & $321(20.4)$ & $85(22.9)$ & $118(22.7)$ & $30(24.2)$ & 0.480 \\
\hline Urinary tract infection & $152(9.7)$ & $37(10.0)$ & $66(12.7)$ & $9(7.3)$ & 0.155 \\
\hline Bacterial pneumonia & $21(1.3)$ & $8(2.2)$ & $10(1.9)$ & $9(7.6)$ & $<0.001$ \\
\hline Bacteremia & $6(0.4)$ & $4(1.1)$ & $6(1.2)$ & $2(1.6)$ & 0.106 \\
\hline Viral infection & $142(9.0)$ & $37(10.0)$ & $44(8.5)$ & $17(13.7)$ & 0.308 \\
\hline Viral pneumonia & $6(0.4)$ & $2(0.5)$ & $9(1.7)$ & $4(3.2)$ & 0.001 \\
\hline Fungal infection & $11(0.7)$ & $3(0.8)$ & $7(1.3)$ & $2(1.6)$ & 0.451 \\
\hline Pneumocystis jiroveci pneumonia & $8(0.5)$ & $2(0.5)$ & $7(1.3)$ & $4(3.2)$ & 0.005 \\
\hline
\end{tabular}

Using data from a Korean nationwide patient registry, we demonstrated that the combination of DRWRi and ABOi increased the risks of graft loss and patient mortality, whereas neither factor alone increased these risks. Poor graft survival after ABOi-DRWRi LDKT was prominent among patients who experienced BPAR, although the combination of ABOi and DRWRi was not an independent risk factor for BPAR. However, the combination of ABOi and DRWRi was an independent risk factor for pneumonia and infection-related death. 
Although the outcomes of ABOi LDKT have been inconsistent among different studies, patients who undergo ABOi LDKT have better long-term survival than those who wait for an extended period of time for an $\mathrm{ABOc}$ transplant from a deceased donor [11]. Furthermore, we recently showed that outcomes of $\mathrm{ABOi}$ LDKT were comparable to those of $\mathrm{ABOc}$ LDKT and better than those of KT from a deceased donor, even in older patients [22]. These findings suggest that ABOi LDKT should be considered an essential treatment option for patients with ESRD when an ABOc donor is not available. In this context, it is important to identify risk factors that affect the outcome of ABOi LDKT.

$\mathrm{ABOi}$ alone was not an independent risk factor for death-censored graft survival in our population, in contrast to the findings of a recent meta-analysis [10]. One reason for this discrepancy might be that overall LDKT outcomes have improved due to the use of tacrolimus-based immunosuppression in the study era. Only $2 \%$ of the patients in our study lost their renal allograft during a mean follow-up of approximately 3 years, a much lower rate than in most studies included in the meta-analysis. This excellent outcome was also influenced by our exclusion of HLA incompatible LDKT from the study population, except for XM-DSA+, which was reported to not increase the short-to-medium-term risk of graft loss [23]. Another possible cause for the good outcomes in our study was the relatively high frequency of rituximab use among the patients who underwent ABOi LDKT. In the previous meta-analysis, ABOi LDKT with rituximab-based desensitization resulted in equal graft survival compared to ABOc LDKT. Rituximab was shown to reduce BPAR in immunologically high-risk patients in a prior randomized controlled study [24]. In Korea, rituximab for pre-transplant desensitization is covered by the national health insurance, so most recipients in ABOi LDKTs are treated with rituximab prior to surgery $[5,7,25]$. Although data regarding rituximab use were available for only $45 \%$ of the patients in our study who underwent ABOi LDKT, $94.8 \%$ of these patients received rituximab before KT, which might explain the high rate of graft survival.

Among the ABOi LDKT patients in our study, only those with DRWRi had lower death-censored graft survival rates compared with ABOc LDKT patients. Donor-recipient size mismatch is a well-known risk factor in KT [26,27]; however, the risk due to size mismatch alone is low (increased risk by approximately 1.1 to 1.2 times) $[16,17]$ or even insignificant in studies of deceased donor KT in the modern era [28]. Therefore, studies have focused on the combination of size mismatch and other mild-to-moderate risk factors, such as extended donor criteria and sex mismatch. The risk of DRWRi was included in the living kidney donor profile index developed in the United States, but it only reflects an increased risk if the DRWR is $<0.9$ [29]. In our study, DRWRi was only a marginal risk factor for death-censored graft survival in the multivariable analysis $(p=0.074)$, which could be due to the relatively small population and the short-to-medium duration of follow-up. Regardless, our results showed that DRWRi had a greater effect on graft survival in ABOi LDKT than in ABOc LDKT.

In the Kaplan-Meier analysis, patients without BPAR events during follow-up had equal graft survival regardless of $\mathrm{ABOi}$ or DRWRi. In contrast, among patients who experienced BPAR, the combination of ABOi and DRWRi was associated with lower deathcensored graft survival, although the difference was not significant, probably because of the small sample size. The low graft survival after ABOi-DRWRi LDKT among patients who experienced BPAR might be due to low functional reserves in relatively small renal allografts, which were well demonstrated in a previous study [18]. More potent rejection, such as antibody mediated rejection due to $\mathrm{ABOi}$, is another possible explanation of the low graft survival after ABOi-DRWRi KT among patients with BPAR. Although our study did not identify different types of acute rejection, our results suggest that the consequences of rejection might be more hazardous in ABOi-DRWRi KT than in KT without both risk factors.

Interestingly, $\mathrm{ABOi}-\mathrm{DRWRi}$ was the independent risk factor for patient survival while ABOi alone was not a significant risk factor in this study. High recipient BMI, which is characteristic of DRWRi, might affect patient survival; however, DRWRi alone did not significantly increase the risk of mortality. In fact, recent studies in the United States found 
that recipient BMI was not an independent risk factor for patient survival after KT [30]. A possible explanation for increased risk of patient death in ABOi-DRWRi KT is a higher rate of severe infection due to pre-transplant desensitization and treatment for BPAR. The ABOi-DRWRi group included many cases in which male patients received kidneys from wives with comparatively low BMI and incompatible blood types, so unrelated donors with relatively low levels of HLA match were frequent in that group. Despite an improved understanding of DSA and the development of immunosuppression, the level of HLA matching is still considered a strong risk factor for BPAR and graft survival [31]. In our study, although the difference was not significant in the multivariable analysis, the ABOi-DRWRi group had a higher rate of BPAR than the other groups in the KaplanMeier analysis. We hypothesize that immunological treatment for BPAR synergized with pre-transplant desensitization for ABOi and resulted in a higher rate of severe infections. This complication might have been further affected by poor renal function due to DRWRi. ABOi alone was also an independent risk factor for pneumonia, although its HR (2.02) was lower than that of ABOi-DRWRi (2.94). The higher rate of mortality due to infection in the ABOi-DRWRi group than the other groups supports our hypothesis, although this finding should be interpreted cautiously because of the small number of events observed.

Our study had some limitations. Because of the short-to-medium duration of followup, our results do not represent the long-term consequences of ABOi and DRWRi. The small sample size of the ABOi-DRWRi group was another limitation, which resulted in large confidence intervals of hazard for some outcomes. Additionally, detailed information, including the strength of anti-blood group antibody and desensitization protocols, was not included in the risk factor analysis because of data unavailability in the registry. Furthermore, potential confounders not controlled by the multivariable analysis because of the small number of events could be present, although we attempted to optimize the adjusted model to obtain statistical relevance.

\section{Conclusions}

The combination of ABOi and DRWRi was an independent risk factor for graft and patient survival after LDKT, whereas neither factor alone significantly affected graft or patient survival. Thus, donor-recipient weight matching should be considered when selecting donors for ABOi LDKT.

Supplementary Materials: The following are available online at https:/ / www.mdpi.com/article/ $10.3390 / j \mathrm{~cm} 10235674 / \mathrm{s} 1$, Figure S1. Distribution of donor-recipient weight ratio; Figure S2. Causes of graft failure in each group; Figure S3. Causes of death in each group; Table S1. Details of ABOi LDKT (for available data only); Table S2. Full results of one-way ANOVA with post hoc analyses for comparison eGFR at each time point; Table S3. Cox regression analysis for death-censored graft survival; Table S4. Cox regression analysis for patient survival; Table S5. Cox regression analysis for BPAR; Table S6. Cox regression analysis for pneumonia.

Author Contributions: D.G.K. had full access to all aspects of the study and takes responsibility for the integrity of the data and the accuracy of the data analysis. D.G.K. and J.Y.L. participated in the research design. S.H.K., K.H.J., K.Y.C., H.R.C., J.L., K.H.H., J.Y. and M.S.K. participated in the performance of the research. J.Y.L. and S.H.C. participated in the data acquisition. D.G.K., S.H.K. and S.H.C. participated in the statistical analysis. J.Y.L. and S.H.C. participated in the writing of the paper. D.G.K. supervised the study process. All authors have read and agreed to the published version of the manuscript.

Funding: This research was supported by grants (2014-ER6301-00, 2014-ER6301-01, 2014-ER6301-02, 2017-ER6301-00, 2017-ER6301-01, 2017-ER6301-02) from the Research of Korea Disease Control and Prevention Agency.

Institutional Review Board Statement: This study was conducted in accordance with the tenets of the Declaration of Helsinki and the Declaration of Istanbul. The protocol was approved by the Yonsei University Wonju College of Medicine (no: CR319359). 
Informed Consent Statement: This study was performed with an exemption for informed consent because of the retrospective nature of the study.

Data Availability Statement: This national registry data cannot be made available to other researchers according to the policy.

Acknowledgments: The Korean Organ Transplantation Registry Study Group; Myoung Soo Kim, Jaeseok Yang, Jin Min Kong, Oh Jung Kwon, Deok Gie Kim, Cheol Woong Jung, Yeong Hoon Kim, Joong Kyung Kim, Chan-Duck Kim, Ji Won Min, Sung Kwang Park, Yeon Ho Park, Jae Berm Park, Jung Hwan Park, Jong-Won Park, Tae Hyun Ban, Sang Heon Song, Seung Hwan Song, Ho Sik Shin, Chul Woo Yang, Hye Eun Yoon, Kang Wook Lee, Dong Ryeol Lee, Dong Won Lee, Sam Yeol Lee, Sang-Ho Lee, Su Hyung Lee, Yu Ho Lee, Jung Pyo Lee, Jeong-Hoon Lee, Jin Seok Jeon, Heungman Jun, Kyung Hwan Jeong, Ku Yong Chung, Hong Rae Cho, Ju Man Ki, Dong-Wan Chae, Soo Jin Na Choi, Sung Shin, Seungyeup Han and Kyu Ha Huh.

Conflicts of Interest: All authors declare no conflict of interest.

\section{References}

1. Tonelli, M.; Wiebe, N.; Knoll, G.; Bello, A.; Browne, S.; Jadhav, D.; Klarenbach, S.; Gill, J. Systematic review: Kidney transplantation compared with dialysis in clinically relevant outcomes. Am. J. Transplant. 2011, 11, 2093-2109. [CrossRef]

2. Takahashi, K.; Saito, K.; Takahara, S.; Okuyama, A.; Tanabe, K.; Toma, H.; Uchida, K.; Hasegawa, A.; Yoshimura, N.; Kamiryo, Y.; et al. Excellent long-term outcome of ABO-incompatible living donor kidney transplantation in Japan. Am. J. Transplant. 2004, 4, 1089-1096. [CrossRef]

3. Speer, C.; Kälble, F.; Nusshag, C.; da Silva, L.P.; Schaier, M.; Becker, L.E.; Klein, K.; Sommerer, C.; Beimler, J.; Leo, A.; et al. Outcomes and complications following ABO-incompatible kidney transplantation performed after desensitization by semiselective immunoadsorption-A retrospective study. Transpl. Int. 2019, 32, 1286-1296. [CrossRef] [PubMed]

4. De Weerd, A.E.; Betjes, M.G.H. ABO-incompatible kidney transplant outcomes: A meta-analysis. Clin. J. Am. Soc. Nephrol. 2018, 13, 1234. [CrossRef] [PubMed]

5. Song, S.H.; Lee, J.; Kim, B.S.; Kim, S.; Lee, J.G.; Jeong, H.J.; Kim, Y.S.; Kim, M.S.; Kim, H.O.; Kim, S.I.; et al. Successful launch of an ABO-incompatible kidney transplantation program to overcome the shortage of compatible living donors: Experience at a single center. Clin. Nephrol. 2017, 88, 117-123. [CrossRef] [PubMed]

6. Opelz, G.; Morath, C.; Süsal, C.; Tran, T.H.; Zeier, M.; Döhler, B. Three-year outcomes following 1420 ABO-incompatible living-donor kidney transplants performed after ABO antibody reduction: Results from 101 centers. Transplantation 2015, 99, 400. [CrossRef]

7. Kong, J.M.; Ahn, J.; Park, J.B.; Chung, B.-H.; Yang, J.; Kim, J.K.; Huh, K.H.; Kim, J.M. ABO incompatible living donor kidney transplantation in Korea: Highly uniform protocols and good medium-term outcome. Clin. Transplant. 2013, 27, 875-881. [CrossRef] [PubMed]

8. Montgomery, J.R.; Berger, J.C.; Warren, D.S.; James, N.T.; Montgomery, R.A.; Segev, D.L. Outcomes of ABO-incompatible kidney transplantation in the United States. Transplantation 2012, 93, 603. [CrossRef] [PubMed]

9. Genberg, H.; Kumlien, G.; Wennberg, L.; Tyden, G. The efficacy of antigen-specific immunoadsorption and rebound of anti-A/B antibodies in ABO-incompatible kidney transplantation. Nephrol. Dial. Transplant. 2011, 26, 2394. [CrossRef] [PubMed]

10. Scurt, F.G.; Ewert, L.; Mertens, P.R.; Haller, H.; Schmidt, B.M.W.; Chatzikyrkou, C. Clinical outcomes after ABO-incompatible renal transplantation: A systematic review and meta-analysis. Lancet 2019, 393, 2059-2072. [CrossRef]

11. Massie, A.B.; Orandi, B.J.; Waldram, M.M.; Luo, X.; Nguyen, A.Q.; Montgomery, R.A.; Lentine, K.L.; Segev, D.L. Impact of ABO-incompatible living donor kidney transplantation on patient survival. Am. J. Kidney Dis. 2020, 76, 616-623. [CrossRef] [PubMed]

12. Luyckx, V.A.; Brenner, B.M. The clinical importance of nephron mass. J. Am. Soc. Nephrol. 2010, 21, 898-910. [CrossRef]

13. Azuma, H.; Nadeau, K.; Mackenzie, H.S.; Brenner, B.M.; Tilney, N.L. Nephron mass modulates the hemodynamic, cellular, and molecular response of the rat renal allograft1. Transplantation 1997, 63, 519-528. [CrossRef]

14. Brenner, B.M.; Milford, E.L. Nephron underdosing: A programmed cause of chronic renal allograft failure. Am. J. Kidney Dis. 1993, 21, 66-72. [CrossRef]

15. McNamara, B.; Diouf, B.; Hughson, M.; Hoy, W.; Bertram, J.F. Associations between age, body size and nephron number with individual glomerular volumes in urban West African males. Nephrol. Dial. Transplant. 2009, 24, 1500-1506. [CrossRef]

16. Miller, A.J.; Kiberd, B.A.; Alwayn, I.P.; Odutayo, A.; Tennankore, K.K. Donor-recipient weight and sex mismatch and the risk of graft loss in renal transplantation. Clin. J. Am. Soc. Nephrol. 2017, 12, 669-676. [CrossRef] [PubMed]

17. Goldberg, R.J.; Smits, G.; Wiseman, A.C. Long-term impact of donor-recipient size mismatching in deceased donor kidney transplantation and in expanded criteria donor recipients. Transplantation 2010, 90, 867-874. [CrossRef]

18. Giral, M.; Foucher, Y.; Karam, G.; Labrune, Y.; Kessler, M.; de Ligny, B.H.; Büchler, M.; Bayle, F.; Meyer, C.; Trehet, N.; et al. Kidney and recipient weight incompatibility reduces long-term graft survival. J. Am. Soc. Nephrol. 2010, 21, 1022-1029. [CrossRef] 
19. Kim, Y.S.; Moon, J.I.; Kim, D.K.; Kim, S.I.; Park, K. Ratio of donor kidney weight to recipient bodyweight as an index of graft function. Lancet 2001, 357, 1180-1181. [CrossRef]

20. Yang, J.; Jeong, J.C.; Lee, J.; Kim, Y.H.; Paik, H.C.; Kim, J.-J.; Park, H.-Y.; Kim, M.S.; Ahn, C. Design and methods of the Korean organ transplantation registry. Transplant. Direct 2017, 3, e191. [CrossRef]

21. Levey, A.S.; Coresh, J.; Greene, T.; Stevens, L.A.; Zhang, Y.; Hendriksen, S.; Kusek, J.W.; Van Lente, F. Using standardized serum creatinine values in the modification of diet in renal disease study equation for estimating glomerular filtration rate. Ann. Intern. Med. 2006, 145, 247-254. [CrossRef]

22. Kim, D.G.; Lee, J.; Kim, M.S.; Kwon, O.J.; Jung, C.W.; Lee, K.W.; Yang, J.; Ahn, C.; Huh, K.H. The Korean organ transplantation registry study group outcomes of ABO-incompatible kidney transplantation in older patients: A national cohort study. Transpl. Int. 2021, 34, 290-301. [CrossRef] [PubMed]

23. Buttigieg, J.; Ali, H.; Sharma, A.; Halawa, A. Positive Luminex and negative flow cytometry in kidney transplantation: A systematic review and meta-analysis. Nephrol. Dial. Transplant. 2018, 34, 1950-1960. [CrossRef] [PubMed]

24. Van den Hoogen, M.W.; Kamburova, E.G.; Baas, M.C.; Steenbergen, E.J.; Florquin, S.M.; Koenen, H.J.P.; Hilbrands, L.B. Rituximab as induction therapy after renal transplantation: A randomized, double-blind, placebo-controlled study of efficacy and safety. Am. J. Transplant. 2015, 15, 407. [CrossRef]

25. Ko, E.J.; Yu, J.H.; Yang, C.W.; Chung, B.H. The Korean organ transplantation registry study group clinical outcomes of ABO- and HLA-incompatible kidney transplantation: A nationwide cohort study. Transpl. Int. 2017, 30, 1215-1225. [CrossRef] [PubMed]

26. El-Agroudy, A.E.; Hassan, N.A.; Bakr, M.A.; Foda, M.A.; Shokeir, A.A.; Shehab el-Dein, A.B. Effect of donor/recipient body weight mismatch on patient and graft outcome in living-donor kidney transplantation. Am. J. Nephrol. 2003, 23, 294. [CrossRef] [PubMed]

27. Kasiske, B.L.; Snyder, J.J.; Gilbertson, D. Inadequate donor size in cadaver kidney transplantation. J. Am. Soc. Nephrol. 2002, 13, 2152-2159. [CrossRef]

28. Arshad, A.; Hodson, J.; Chappelow, I.; Nath, J.; Sharif, A. The influence of donor to recipient size matching on kidney transplant outcomes. Transplant. Direct 2018, 4, e391. [CrossRef] [PubMed]

29. Massie, A.B.; Leanza, J.; Fahmy, L.M.; Chow, E.; Desai, N.M.; Luo, X.; King, E.A.; Bowring, M.; Segev, D.L. A risk index for living donor kidney transplantation. Am. J. Transplant. 2016, 16, 2077-2084. [CrossRef]

30. Sureshkumar, K.K.; Chopra, B.; Josephson, M.A.; Shah, P.B.; McGill, R.L. Recipient obesity and kidney transplant outcomes: A mate-kidney analysis. Am. J. Kidney Dis. 2021, 78, 501-510.e1. [CrossRef]

31. Yacoub, R.; Nadkarni, G.N.; Cravedi, P.; He, J.C.; Delaney, V.B.; Kent, R.; Chauhan, K.N.; Coca, S.G.; Florman, S.S.; Heeger, P.S.; et al. Analysis of OPTN/UNOS registry suggests the number of HLA matches and not mismatches is a stronger independent predictor of kidney transplant survival. Kidney Int. 2018, 93, 482-490. [CrossRef] [PubMed] 